\title{
Antioxidant Activity and Total Phenolic Content of Sesame (Sesamum indicum L.) Seed Oil
}

\author{
D. Bopitiya and T. Madhujith ${ }^{1^{*}}$ \\ Postgraduate Institute of Science, \\ University of Peradeniya \\ Sri Lanka
}

\begin{abstract}
Minor components, especially antioxidative constituents, play an imperative role in the nutritional and health impact of edible oils. This study was carried out to determine the antioxidant activity and total phenolic content of Sesame (Sesamum indicum L.) seed oil, which currently remains as an underutilized oil in Sri Lanka. The phenolic fraction of the oil was extracted into methanol by passing the oil through a glass column containing silica $(60$ A). The Total Phenolic Content (TPC) was determined using FolinCiocalteu's colorimetric method and expressed as mg of gallic acid equivalents (GAE) per gram of extract. The antioxidant potential of the oil extracts was assessed using DPPH and ABTS radical scavenging assays and $\beta$-carotene/linoleate model system. $\alpha$-Tocopherol was used as the reference antioxidant. Sesame seed oil extracts possessed a higher TPC (26.00 \pm $0.14 \mathrm{mg} \mathrm{GAE} / \mathrm{g}$ of extract) compared to the reference antioxidant, $\alpha$-tocopherol (18.00 \pm $0.12 \mathrm{mg} G A E / g$ ). The DPPH radical scavenging activity of the oil extract and $\alpha$-tocopherol was $0.026 \mathrm{mg} / \mathrm{mL}$ and $0.031 \mathrm{mg} / \mathrm{mL}$, respectively. The ABTS radical scavenging activity of the oil extract and $\alpha$-tocopherol were $58.0 \%$ and $46.0 \%$, respectively, at $2.0 \%(w / v)$ concentration. Sesame seed oil extract exhibited a significantly higher percentage $(p<0.05)$ of inhibition of linoleic acid induced oxidation of $\beta$-carotene (53.6\%) compared to $\alpha$ tocopherol $(45.0 \%)$ at $2.0 \%(w / v)$ concentration. Results revealed that the sesame seed oil possessed a strong antioxidant activity compared to $\alpha$-tocopherol, therefore, can be categorized as an edible oil with a high potential of antioxidant activity.
\end{abstract}

Keywords: Antioxidant, sesame seed oil, total antioxidant capacity, total phenoilc content

\section{INTRODUCTION}

Sesame (Sesamum indicum L.) oil, also known as gingelly oil or til oil, is a highly unsaturated edible oil rich in essential fatty acids such as linoleic acid. Sesame has an annual production of less than 1 MMT and cultivated mainly in India, China, Myanmar, Sudan, Mexico and Egypt. Sesame seed is rich in oil carrying nearly $40-60 \%$ of oil. Sesame oil contains almost equal levels of oleic (35 to 54\%) and linoleic (39 to 59\%), 10\% of palmitic acid and $5 \%$ of stearic acid (Hall, 2003). It is also rich in various bioactive compounds including phytosterols, tocopherols and lignans such as sesamin, sesamolin and sesaminol, which are known to play an important role in providing stability against oxidation of oil and contribute to antioxidative activity (Shahidi et al., 1997; Philip et al., 2010). Sesamin and

\footnotetext{
Department of Food Science and Technology, Faculty of Agriculture, University of Peradeniya, Sri Lanka
}

Corresponding author: madujith@yahoo.com 
sesaminol are converted to more powerful antioxidative constituent, sesamol when subjected to high temperatures. Thus, the presence of sesamin and sesaminol is favoured in frying oils as sesamol provides a protective action against autoxidation of oil. Several studies have reported that sesame oil contributes to prevent various disorders such as hypertension, hypercholesterolemia, cancer and aging (Philip et al., 2010). Moreover, sesame oil exhibits multiple physiological functions such as decreasing plasma triacylglycerol and arachidonic acid levels, imparting anti-inflammatory and estrogenic activities (Shahidi et al., 1997; Hemalatha et al., 2007; Philip et al., 2010). Sesame seed oil shows an exceptionally high oxidative stability compared to soybean, corn and most other popular vegetable oils (Shahidi et al., 1997; Carrasco-pancorbo et al., 2005; Katerina et al., 2010). Although sesame oil is used by some ethnic groups, it still remains as an underutilized oil in many parts of the world including Sri Lanka.

Even though oils provide essential nutrients such as essential fatty acids and fat soluble vitamins, many epidemiological studies report that the over consumption of edible oils lead to non communicable diseases (Shahidi et al., 1997; Philip et al., 2010). Moreover, edible oils can easily become oxidized during processing and storage, thus producing a plethora of oxidative products which bring about oxidative stress-associated disease conditions such as atherosclerosis, diabetes mellitus, rheumatoid arthritis, cancer, aging and neurodegenerative diseases including Alzheimer's disease (Shahidi et al., 1997; Carrasco-pancorbo et al., 2005; Alessandra et al., 2006; Katerina et al., 2010).

Edible oils which are rich in unsaturated fatty acids especially, monounsaturated fatty acids (MUFA) are known to lower total and LDL cholesterol, triglycerides, increase HDL cholesterol, help controlling blood pressure, impart antithrombotic properties and help improve insulin sensitivity (Alessandra et al., 2007). In addition, MUFA increase the stability of edible oils. Presence of bioactive components especially, phenolic antioxidants are attributable to better oxidative stability of oils. Moreover, the bioactive components present in oils may serve as important bioactives that help reducing the oxidative stress in the human body (Shahidi et al., 1997; Carrasco-pancorbo et al., 2005; Katerina et al., 2010). In addition, the minor components present in edible oils contribute to improve texture and palatability and add characteristic flavours to food preparations (Shahidi et al., 1997).

Over the recent years, much attention has been focused on the edible oils which exhibit high oxidative stability. Despite the high content of MUFA, sesame oil remains as an underutilized oil in Sri Lanka. The studies on bioactive compounds in sesame oil are extremely scarce. In this backdrop, the present study was carried out to quantify the total phenoilc content and evaluate the antioxidant potential of sesame oil.

\section{METHODOLOGY}

Sesame oil was procured from a sesame oil mill located in Kekirawa, Anuradhapura. 2,2diphenyl-1-picrylhydrazylhydrate (DPPH), Folin-Ciocalteu's phenol reagent, gallic acid, 2,2'-azinobis (3-ethylbenzothiazoline-6-sulfonic acid) diammonium salt (ABTS), linoleic acid, $\beta$-carotene, and Tween 20 were purchased from Sigma Chemicals Company, USA. All other chemicals used were analytical grade and obtained from either Sigma, USA or Himedia, India. 


\section{Extraction of phenolic constituents}

Phenolic fraction present in fifty gram of oil was extracted into methanol by passing the oil through a glass column packed with silica $(60-\AA$ pore diameter) according to the method described by Steel et al. (2005). Hexane and methanol (1:1) mixture was used for conditioning the column and hexane and ethyl acetate (9:1) mixture was used for washing the column. The oil sample was introduced dissolved in hexane and the phenolic fraction was extracted into methanol. The extract was recovered after desolventizing in vacuo at $40^{\circ} \mathrm{C}$. The so prepared extracts were stored under frozen condition $\left(-20^{\circ} \mathrm{C}\right)$ after flushing with nitrogen. The frozen extracts were thawed and appropriately diluted before chemical analysis.

\section{Determination of total phenolic content (TPC)}

Total phenolic content was determined using Folin-Ciocalteu's colorimetric method and expressed as mg of gallic acid equivalents (GAE) per gram extract (Aleksander et al., 2008).

\section{Assessment of antioxidant activity of extracts}

Antioxidant potential of the oil extract was measured using DPPH and ABTS radical scavenging assays and $\beta$-carotene/linoleate model system. $\alpha$-Tocopherol was used as the reference antioxidant. Results of the DPPH and ABTS radical scavenging assays were expressed as $\mathrm{IC}_{50}$ value and percentage discoloration, respectively. The dose dependence behavior of the extract towards DPPH and ABTS radicals was also determined by using varying quantities $(0.1-0.5 \mathrm{~g})$ of the extract (Madhujith and Shahidi, $2006 \& 2009)$. The antioxidant activity as measured by $\beta$-carotene/linoleate model system was calculated as percentage discoloration (Madhujith et al., 2004). Data were analyzed using Ms Office (Excel 2007) and MINITAB (version 16) software.

\section{RESULTS AND DISCUSSION}

The total phenolic content (TPC) of the methanolic sesame oil extracts was $26.00 \pm 0.14 \mathrm{mg}$ GAE/g of extract. Sesame oil extract contained higher TPC compared to other commonly available vegetable oils. TPC of sunflower, corn, rapeseed, and soy oils are 12.0, 12.6, 13.1, and 14.8mg GAE /g of methanolic extract, respectively (Aleksander et al., 2008).

The DPPH radical scavenging activity expressed as $\mathrm{IC}_{50}$ value of the sesame oil extract and $\alpha$-tocopherol was $0.026 \mathrm{mg} / \mathrm{mL}$ and $0.031 \mathrm{mg} / \mathrm{mL}$, respectively. $\mathrm{IC}_{50}$ value is inversely proportional to the antioxidant activity. According to the $\mathrm{IC}_{50}$ value, sesame seed oil can be categorized as an oil with considerable antioxidant potential. Studies reported that the $\mathrm{IC}_{50}$ value of selected vegetable oil extracts, namely soy, sunflower, rapeseed, corn ranged from 29.7 to $34.0 \mu \mathrm{g} / \mathrm{mL}$ (Aleksander et al., 2008).

The ABTS radical scavenging activity expressed as percentage discoloration at $2.0 \%(\mathrm{w} / \mathrm{v})$ concentration of the oil extract and $\alpha$-tocopherol was $58.0 \%$ and $46.0 \%$, respectively. Recent studies reported that the percentage inhibition of extracts obtained from selected vegetable oils, namely soy, sunflower, rapeseed and corn oils ranged from 11.1 to $51.2 \%$ (Aleksander et al., 2008). The reaction between the extracts and $\mathrm{ABTS}^{\cdot+}$ was not complete within six minutes of reaction ( 0.742 to 0.157 of absorbance) (Fig 1). The ABTS radical scavenging 
efficacy of sesame oil extract was stronger than that of the reference antioxidant, $\alpha$ tocopherol. Based on the percentage inhibition of ABTS radical by the extracts, sesame oil can be categorized as an oil with considerable antioxidant potential.

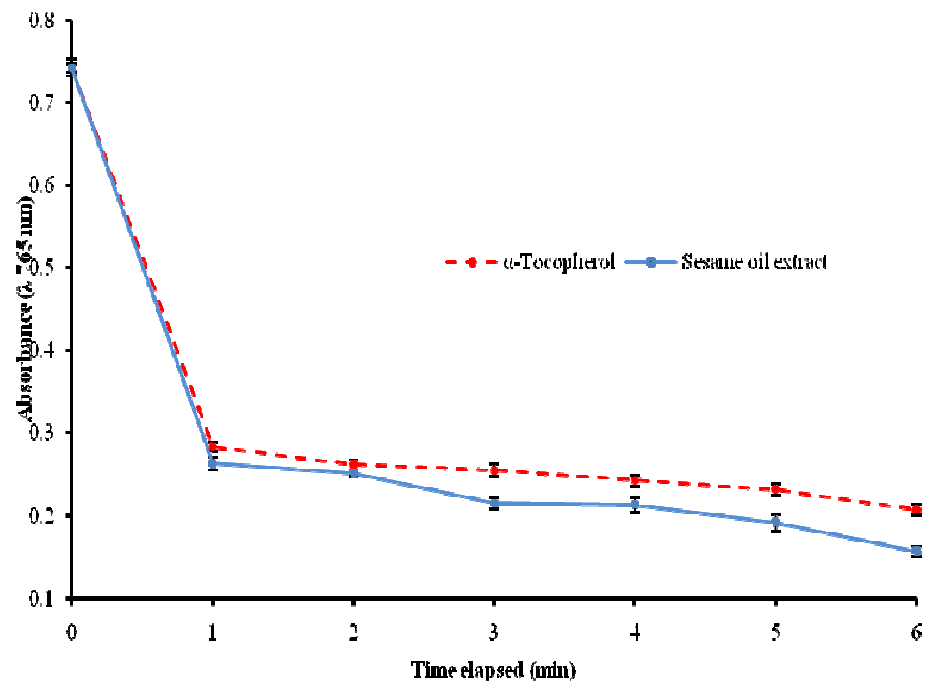

Fig. 1. The change of absorbance of the reaction mixture of $\mathrm{ABTS}^{\circ+}$ and extracts or $\alpha$-tocopherol over six minutes

$\beta$-Carotene is a compound highly vulnerable for autoxidation. The $\beta$-carotene/linoleate model system has been used as an important assay to assess the efficacy of antioxidative constituents in inhibition of linoleic acid induced oxidation of $\beta$-carotene. Sesame oil extracts were capable of effectively retarding the oxidation of $\beta$-carotene in the emulsion system compared to the reference antioxidant, $\alpha$-tocopherol (Fig 2). The inhibition of degradation of $\beta$-carotene as expressed by percentage of discoloration of sesame oil extract and $\alpha$-tocopherol were $53.58 \%$ and $45.03 \%$, respectively, at $2.0 \%(\mathrm{w} / \mathrm{v})$ concentration. 


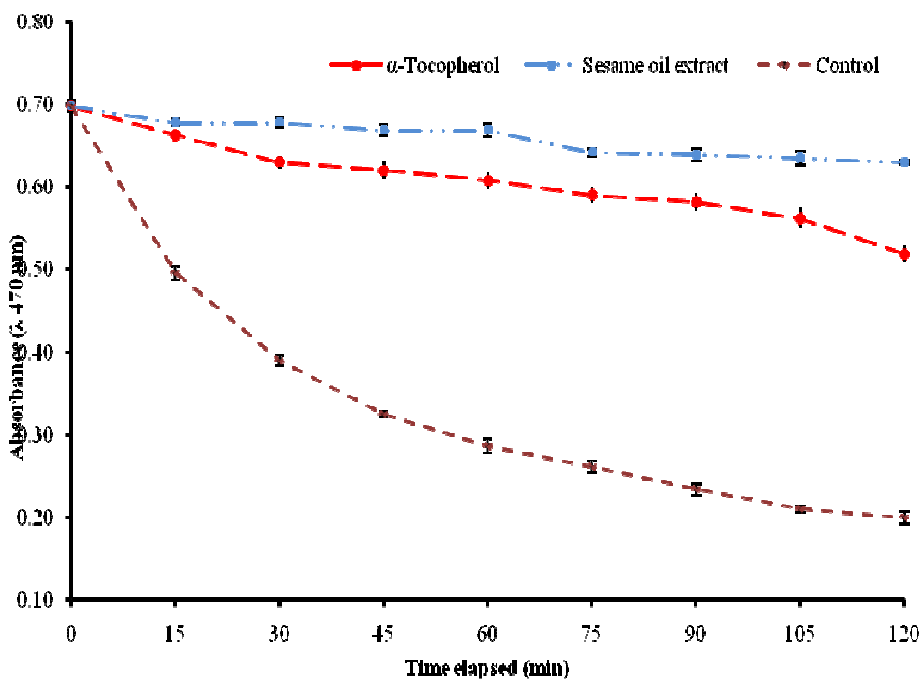

Fig. 2. Decrease of absorbance in the emulsion system containing $\beta$-carotene-linoleic acid-extract or reference antioxidant over 120 minutes

\section{Dose dependence behavior of antioxidative extracts}

It was observed that the antioxidant efficacy of the extract as measured by DPPH and ABTS radical scavenging assays increased with the quantity of the extract confirming that compounds present in the extract contribute to the antioxidant activity of the oil (Fig 3).

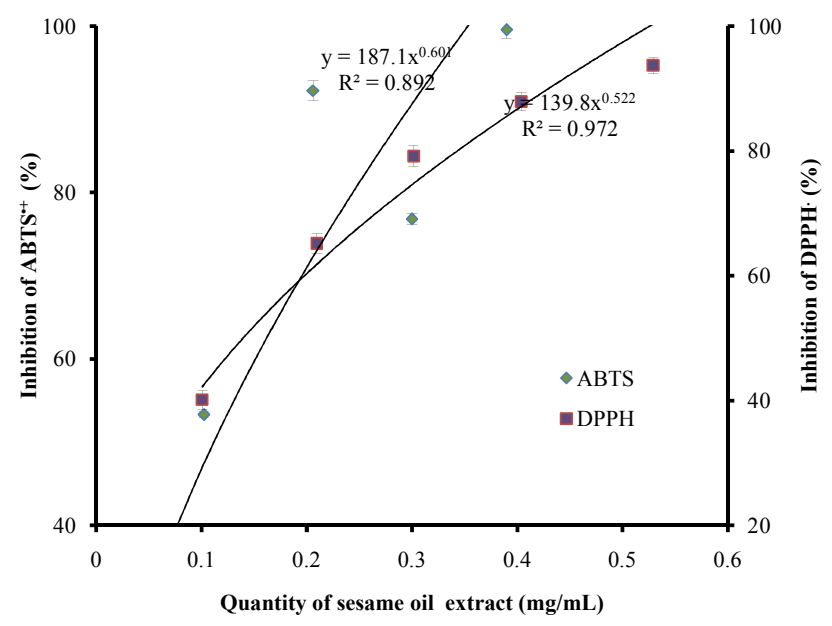

Fig. 3. Correlation between quantity of the oil extract and percentage inhibition of $\mathrm{ABTS}^{\circ+}$ and DPPH 


\section{CONCLUSIONS}

Sesame seed oil possessed comparatively higher quantity of phenolics. The antioxidant activity of methanolic sesame oil extracts as measured by DPPH and ABTS radical scavenging potential, and inhibition of degradation of $\beta$-carotene was significantly higher than that of the reference antioxidant, $\alpha$-tocopherol. Sesame oil can be categorized as an edible oil with moderately high potential of antioxidant activity. It is evident that the minor compounds present in sesame oil contribute to the antioxidant activity. It can also be concluded that the antioxidant activity as measured by ABTS and DPPH radicals is dose dependant and no prooxidant activity was exhibited even at the highest level tested.

\section{ACKNOWLEDMENT}

The authors thank National Research Council of Sri Lanka (NRC) for the financial support.

\section{REFERENCES}

Alessandra Bendini, Lorenzo Cerretani, Samuele Vecchi, Alegia Carrasco-Pancorbo, and Giovanni Lercker (2006). Protective effects of extra virgin olive oil phenolics on oxidative stability in the presence or absence of copper ions, J. Agric. Food Chem., 54, 4880-4887.

Aleksander, S., Malgorzata, N., and Elenora, L. (2008). The content and antioxidant activity of phenoilc compounds in cold pressed plant oils. Journal of Food Lipids, 15, 137-149.

Carrasco-pancorbo, A., Cerretani, L., Bendini, A., Segura-carrentero, A., Del Carlo, M., Gallina-toschi, T., Lercker, G., Compangnone, D., and Fernaa Ndez-gutiea (2005). Evaluation of the antioxidant capacity of individual phenolic compounds in virgin olive oil. J. Agric. Food Chemistry, 53, 8918-8925.

Hall, C. III, (2003). Sesame seed oil. In Lipids for Functional Foods and Nutraceuticals, Gunstone, F.D. (Ed.) The Oily Press, Bridgewater, UK pp. 83-90.

Hemalatha, S. and Ghafoorunissa (2007). Sesame lignans enhance the thermal stability of edible vegetable oils. Food Chemistry, 105, 1076-1085.

Katerina S. Miniotia and Constantinos A. (2010). Georgioua Comparison of different tests used in mapping the Greek virgin olive oil production for the determination of its total antioxidant capacity. Grasa Y Aceites, 61(1), 45-51.

Madhujith, T., Marian Naczk and Shahidi, F. (2004). Antioxidant activity of pea beans (Phaseolus vulgaris L.) J. Food Lipids, 11(3), 220.

Madhujith, T. and Shahidi, F. (2006). Optimization of extracting antioxidative constituents of six barley cultivars and their antioxidant properties J. of Agric. Food Chem., 54(21), 80488057.

Madhujith, T. and Shahidi, F. (2009). Antioxidant potential of barley as affected by alkaline hydrolysis and release of insoluble-bound phenolics. Food Chemistry 117,615-620. 
Philip John Kanu, Joseph Zed Bahsoon, Jestina Baby Kanu, and Joseph BA Kandeh (2010). Nutraceutical importance of sesame seed and oil: A review of the contribution of their lignans. Journal of Biomedical Research, 2(1), 4-16.

Shahidi, F., Wanasundara, P.K.J.P.D., and Wanasundara, U.N. (1997). Changes in edible fats and oils during processing. Journal of Food Lipids, 4(19), 9-23.

Steel C.J., Dobarganes M.C., Barrera-Arellano D. (2005). The Influence of natural tocopherols during thermal oxidation of refined and partially hydrogenated soybean oils. Grasas Aceites, 56, 46-52. 